Trauma Berufskrankh 2012 • 14[Suppl 2]:147-148 DOI 10.1007/s10039-011-1817-x

Online publiziert: 8. März 2012

(c) Springer-Verlag 2012
T. Malalla ${ }^{1} \cdot$ S. Reichel ${ }^{2}$

${ }^{1}$ Unfallbehandlungsstelle Berlin

${ }^{2}$ Berufsgenossenschaft der Bauwirtschaft, Berlin

\title{
Netzwerkpartner Arbeitstherapeut im Rehabilitationsmanagement
}

Die Unfallbehandlungsstelle Berlin (UBS) ist das ambulante medizinische Kompetenzzentrum der Berufsgenossenschaften (BG) für Berlin und dessen Umland. Zum umfangreichen Leistungsangebot der UBS gehören die ambulante Unfallversorgung, die Umsetzung des BG-Heilverfahrens, Begutachtungen und moderne Therapieleistungen wie die erweiterte ambulante Physiotherapie nach Arbeits-, Schul- und Wegeunfällen. Umfassende Angebote in Krankengymnastik, Physiotherapie, medizinischer Trainingstherapie, Arbeitstherapie und Gesundheitsförderung ergänzen das Leistungsspektrum.

In Kooperation mit dem Unfallkrankenhaus Berlin bietet die UBS außerdem verschiedene Spezialsprechstunden an, die eine individuell auf den Patienten abgestimmte Diagnostik und Therapie ermöglichen. Ein erfahrenes Team aus Ärzten, Physiotherapeuten und Sportlehrern sorgt dabei für optimale Behandlungsergebnisse und hohe Versorgungsqualität.

\section{Arbeitstherapie}

Im Rahmen der Arbeitstherapie fließen so frühzeitig wie möglich arbeits- und berufsbezogene Maßnahmen in die medizinische Rehabilitation mit ein. Dadurch wird der Versicherte stufenweise durch konkrete, arbeitsplatzrelevante Tätigkeiten unter arbeitsplatznahen Bedingungen auf seine schnellstmögliche Rückkehr ins Berufsleben vorbereitet. Ziel ist die Adaptation berufsspezifischer Arbeitsschritte in 3 Phasen.
Phase 1 - motorisches Training. In der ersten Phase der berufsspezifischen Arbeitstherapie steht das Erlangen von Grundfertigkeiten der Hand und der oberen Extremität im Vordergrund. Die Ergotherapie erfolgt in Form von Einzelbehandlungen motorisch-funktionell oder als begleitende Therapie zur erweiterten ambulanten Physiotherapie. Der Arbeitstherapeut führt den Patienten in dieser Phase Schritt für Schritt an arbeitsrelevante Tätigkeiten heran.

Eingeleitet wird Phase 1 durch den behandelnden Arzt. Bedarfsgerecht findet ein erstes Gespräch mit dem Rehabilitationsmanager der Berufsgenossenschaft, dem Durchgangsarzt und dem Arbeitstherapeuten statt. Am Ende der Phase 1 erfolgt eine Einschätzung der bisherigen motorischen Fähigkeiten durch den Arzt und den Arbeitstherapeuten. Der behandelnde Durchgangsarzt leitet entsprechend der erreichten Fähigkeiten die Phase 2 ein.

Phase 2 - berufsspezifisches Training. Hier steht die Verbesserung der motorischen Ausdauer sowie der Koordination und berufsspezifischen Konditionierung im Vordergrund. Zu Beginn erfolgen eine ausführliche Arbeitsplatzanamnese und eine Festlegung der berufsspezifischen Problemstellung (Welche motorischen Defizite sind weiterhin vorhanden? Welche Folgen ergeben sich für den Arbeitsplatz?). Anhand des erstellten Belastungsprofils durchläuft der Patient in Phase 2 täglich ein Training in der Holzwerkstatt sowie ein berufsspezifisches
Übungsprogramm in der medizinischen Trainingstherapie (MTT).

Bedarfsgerecht findet in Phase 2 ein Gespräch mit dem Rehabilitationsmanager, dem Durchgangsarzt und dem Arbeitstherapeuten statt. Sollte eine therapeutisch begleitete Arbeits- und Belastungserprobung (Phase 3 ) angezeigt sein, nehmen der Rehabilitationsmanager oder die UBS mit dem Arbeitgeber bzw. dem Kooperationsbetrieb Kontakt auf. Am Ende der Phase 2 erfolgen ein Abgleich zwischen dem Arbeitsplatzanforderungsund dem aktuellen Fähigkeitsprofil durch den Arzt und den Arbeitstherapeuten sowie ggf. die Überleitung in Phase 3.

Phase 3 - Training am Arbeitsplatz. Der Patient führt berufsrelevante Tätigkeiten direkt am Arbeitsplatz oder in einem entsprechenden Kooperationsbetrieb (z. B. Lehrbauhof der Fachgemeinschaft Bau Berlin und Brandenburg e. V.) durch. Der Prozess der Arbeits- und Belastungserprobung wird vom Arbeitstherapeuten und dem zuständigen Ausbilder begleitet und beurteilt. Als Grundlage dienen die üblichen Leistungsvorgaben des entsprechenden Gewerbes. Die Einschätzung der arbeitsrelevanten Fähigkeiten und die Belastbarkeit des Versicherten sind die Grundlage für das Abschlussgespräch mit dem Rehabilitationsmanager, dem Durchgangsarzt und dem Arbeitstherapeuten. Ziel der Phase 3 ist die Wiedererlangung der Arbeitsfähigkeit des Patienten in seiner zum Unfallzeitpunkt ausgeübten Tätigkeit. 
Die Unfallbehandlungsstelle Berlin entwickelte sich mit diesem individuell auf den Patienten abgestimmten Stufenmodell zum Netzwerkpartner für alle Berufsgenossenschaften im Großraum Berlin. Kurze interdisziplinäre Wege zwischen dem Unfallkrankenhaus Berlin (ukb), der UBS Berlin, den behandelnden Durchgangsärzten sowie den zuständigen Rehabilitationsmanagern der Berufsgenossenschaften ermöglichen es, schnell und gezielt die Therapie zu steuern, um eine möglichst kurzfristige und nachhaltige berufliche Wiedereingliederung des Versicherten zu erreichen. Nach ersten Ergebnissen gelingt diese bei über $80 \%$ der in der Arbeitstherapie der UBS Berlin vorgestellten Versicherten.

\section{Fallbeispiel}

Es verdeutlicht die Effektivität des Einsatzes von Arbeitstherapie als Netzwerkpartner: Der Versicherte S. erlitt als ungelernter Eisenflechter im Alter von 31 Jahren am 15.06.2010 einen Arbeitsunfall, bei welchem er bei einem Sturz auf der Baustelle mit der rechten Hand an einem $\mathrm{Na}$ gel hängenblieb. Am selben Tag wurde stationär eine Komplexverletzung der rechten Hand mit Durchtrennung der oberflächlichen Beugesehne des 3. bis 5 . Fingers sowie der tiefen Beugesehne des 4. Fingers und einer Läsion der palmaren Fingernerven N6-N10 diagnostiziert und mit Beugesehnen- und Nervennähten erstversorgt.

Am 21.06.2010 wurde im Rahmen des Managements für Handverletzungen am ukb eine Vorstellung des Versicherten in der Handsprechstunde der UBS Berlin empfohlen. Ab 06.07.2010 erfolgten engmaschig Vorstellungen in der Handsprechstunde, welche zur Verordnung von täglicher Ergotherapie führten. Noch im August 2010 war die verbliebene Narbe hart und nicht verschiebbar, der beschädigte Nerv nicht gewachsen, sodass eine Neurolyse in Erwägung gezogen werden musste. Eine berufliche Wiedereingliederung als Eisenflechter war laut Bericht vom 05.08.2010 nur schwer vorstellbar. Erst ab Mitte November 2010 zeigte sich eine beginnende Reinnervation. Am 20.12.2010 wurde anlässlich der turnusmäßigen Vorstellung des Versicherten in der Handsprechstunde der UBS Berlin ergänzend zur Ergotherapie 5-mal wöchentlich Arbeitstherapie verordnet. Bei weiteren Vorstellungen am 21.01.2011/18. 02.2011/11.03.2011 waren die positiven Effekte der in der UBS Berlin durchgeführten Arbeitstherapie bereits deutlich feststellbar. Insbesondere war die grobe Kraft in der betroffenen Hand des Versicherten gut aufgebaut worden, und trotz bestehender Sensibilitätsdefizite konnten alle Finger vollständig gestreckt werden. Dieser Unfallfolgezustand ermöglichte dem Versicherten eine Arbeits- und Belastungserprobung (ABE) im (alten) Beruf des Eisenflechters ab 21.02.2011. Nach erfolgreicher Absolvierung der 6-wöchigen $\mathrm{ABE}$ konnte der Versicherte $\mathrm{ab}$ 02.04.2011 seine Tätigkeit wieder vollschichtig aufnehmen. Zum Zeitpunkt der Tätigkeitsaufnahme bestand eine subjektiv gute Sensibilität der verletzten Hand, der Faustschluss war komplett möglich, eine Behandlung nicht mehr erforderlich.

\section{Fazit für die Praxis}

Die 8-wöchige ergänzende Arbeitstherapie in der UBS Berlin trug wesentlich dazu bei, das oben dargestellte optimale Rehabilitationsergebnis zu erzielen. Einen besonderen Benefit für den Versicherten und die zuständige Berufsgenossenschaft stellt hierbei die gelungene Wiedereingliederung in die vor dem Unfall ausgeübte Tätigkeit dar, weil die Erbringung von Leistungen zur Teilhabe am Arbeitsleben an einen ungelernten Eisenflechter für beide Beteiligte sehr aufwendig und kostenintensiv gewesen wäre.

\section{Korrespondenzadresse}

\section{S. Reichel}

Berufsgenossenschaft der Bauwirtschaft, Hildegardstraße 29/30, 10715 Berlin stefan.reichel@bgbau.de

Interessenkonflikt. Der korrespondierende Autor gibt an, dass kein Interessenkonflikt besteht.
Trauma Berufskrankh 2012 - 14:[Suppl 2]147-148

DOI 10.1007/s10039-011-1817-x

(C) Springer-Verlag 2012

T. Malalla.S. Reichel

Netzwerkpartner Arbeitstherapeut im Rehabilitationsmanagement

\section{Zusammenfassung}

Durch gezielte Nutzung des Arbeitstherapieangebots bei einem Netzwerkpartner im Rehabilitationsmanagement lässt sich die Quote der in ihre alte Tätigkeit wieder eingegliederten Versicherten deutlich steigern. Die Unfallbehandlungsstelle Berlin bedient sich im Rahmen ihres Arbeitstherapieangebots eines 3-Phasen-Modells: 1 . motorisches Training, 2. berufsspezifisches Training und 3. Training am Arbeitsplatz.

Schlüsselwörter

Rehabilitation - Rehabilitationsmanagement . Arbeitsplatz · 3 -Phasen-Modell · Training

\section{Occupational therapist as network partner in rehabilitation management}

\section{Abstract}

The targeted use of available resources for occupational therapy offered by a network partner in rehabilitation management makes it possible to considerably increase the proportion of insured persons able to return to their former employment. The outpatient trauma center in Berlin makes use of a threephase model as part of its services offered for occupational therapy: (1) training in motor skills, (2) job-specific training, and (3) training at the workplace.

\section{Keywords}

Rehabilitation · Rehabilitation management . Employment · Three-phase model · Training 